Revue internationale P.M.E.

Économie et gestion de la petite et moyenne entreprise

Revue

internationale

PME

\title{
Les valeurs du dirigeant et la croissance de la PME
}

\section{Véra Ivanaj et Sybil Géhin}

Volume 10, numéro 3-4, 1997

URI : https://id.erudit.org/iderudit/1009031ar

DOI : https://doi.org/10.7202/1009031ar

Aller au sommaire du numéro

Éditeur(s)

Presses de l’Université du Québec

ISSN

0776-5436 (imprimé)

1918-9699 (numérique)

Découvrir la revue

Citer cette note

Ivanaj, V. \& Géhin, S. (1997). Les valeurs du dirigeant et la croissance de la PME. Revue internationale P.M.E., 10(3-4), 81-108. https://doi.org/10.7202/1009031ar

\section{Résumé de l'article}

Cet article présente les résultats d'une étude portant sur les valeurs du dirigeant et la stratégie de croissance de la PME. L'échantillon est issu de 500 entreprises de 100 à 200 salariés dans le secteur de la plasturgie en France (1993). Les données recueillies sont issues des réponses à un questionnaire envoyé par voie postale (taux de réponse 8,5\%). Les résultats obtenus confirment la relation entre les valeurs et la stratégie de croissance et dressent les différents profils de valeurs. Ainsi, les dirigeants qui présentent un ensemble d'attitudes traduisant des sentiments de confiance globale semblent plus disposés à la croissance que les dirigeants centrés sur la sécurité et la protection. La croissance semble liée à un ensemble de valeurs relatives à l'affirmation de soi plutôt qu'à la croyance en Dieu, au sentiment d'accomplissement personnel plutôt qu'à l'intérêt propre du travail, à un climat de détente dans le milieu familial plutôt qu'à une éducation stricte, à une approche ouverte des autres plutôt qu'à la méfiance et à une attitude positive envers l'avenir plutôt qu'à la prudence.
Ce document est protégé par la loi sur le droit d'auteur. L'utilisation des services d'Érudit (y compris la reproduction) est assujettie à sa politique d'utilisation que vous pouvez consulter en ligne.

https://apropos.erudit.org/fr/usagers/politique-dutilisation/ 


\title{
Les valeurs du dirigeant et la croissance de la PME
}

\author{
Véra IVANAJ \\ Sybil GÉHIN \\ Institut commercial de Nancy \\ Université de Nancy II
}

MOTS CLÉS

\section{Valeurs - PME-PMI-Dirigeant - Stratégie de croissance - Industrie de la plasturgie - France}

\begin{abstract}
RÉSUMÉ
Cet article présente les résultats d'une étude portant sur les valeurs du dirigeant et la stratégie de croissance de la PME. L'échantillon est issu de 500 entreprises de 100 à 200 salariés dans le secteur de la plasturgie en France (1993). Les données recueillies sont issues des réponses à un questionnaire envoyé par voie postale (taux de réponse 8,5\%). Les résultats obtenus confirment la relation entre les valeurs et la stratégie de croissance et dressent les différents profils de valeurs. Ainsi, les dirigeants qui présentent un ensemble d'attitudes traduisant des sentiments de confiance globale semblent plus disposés à la croissance que les dirigeants centrés sur la sécurité et la protection. La croissance semble liée à un ensemble de valeurs relatives à l'affirmation de soi plutôt qu'à la croyance en Dieu, au sentiment
\end{abstract}

\section{LES AUTEURS}

Véra Ivanaj est économiste diplômée de l'Université de Tirana (Albanie). Elle appartient au GREGE (Groupe de recherche en gestion) de l'Université de Nancy II. Ses principaux domaines de recherche portent sur la prise de décision et la stratégie d'entreprise.

Sybil Géhin, professeur associée, responsable du Département management des ressources humaines à l'Institut commercial de Nancy (Université de Nancy II), s'intéresse essentiellement au management des hommes dans les organisations.

Adresse : Pôle lorrain de gestion, Institut commercial de Nancy, Université de Nancy II, 13, rue Michel-Ney, C.O. 75, 54037 Nancy Cedex, France 
d'accomplissement personnel plutôt qu'à l'intérêt propre du travail, à un climat de détente dans le milieu familial plutôt qu'à une éducation stricte, à une approche ouverte des autres plutôt qu'à la méfiance et à une attitude positive envers l'avenir plutôt qu'à la prudence.

\begin{abstract}
This article presents the results of a study of values and developmental strategies of small and medium sized firms. The sample is the result of 500 firms, each from 100 to 200 employees, in the plastic-producing sector in France (1993). The collected data come from answers to a questionnaire sent by post (reported answers totalling $8.5 \%)$. The results obtained corroborate the relation between values and developmental strategies and lay out various profiles of values. The directors furnishing a grouping of attitudes explaining feelings of overall confidence seem more predisposed to development than those focusing on security and protection. Development seems related to a grouping of values centered on self-affirmation rather than on the belief in God, on a feeling of personal accomplishment rather than on the intrinsic value of work, on a relaxed atmosphere at home rather than on a strict upbringing, on an open-minded attitude towards others rather than on mistrust, and finally, on a positive attitude vis-à-vis the future rather than on caution.
\end{abstract}

\title{
RESUMEN
}

Este artículo presenta los resultados de un estudio referente a los valores del dirigente y a la estrategía de crecimiento de la PyME. La muestra procede de 500 empresas de 100 a 200 asalariados en el sector de la plasturgia en Francia (1993). Los dados reunidos proceden de respuestas a un cuestionario remitido por correo (tasa de contestación $8,5 \%$ ). Los resultados reunidos confirman la relación entre los valores y la estrategía de crecimiento y establecen los diferentes perfiles de valores. Así piues, los dirigentes que presentan un conjunto de atitudes expresando sentimientos de confianza global parecen más dispuestos al crecimiento que los dirigentes que se dedican a la seguridad y la protección. El crecimiento parece liado a un conjunto de valores relativas a la afirmación de si más bien que en la creencia en Dios, al sentimiento de la realización personal más bien que al interés propio del trabajo, a un ambiente de respiro con la familia más bien que a una educación estricta, más bien que una apertura a los demás más bien que a la desconfianza y a una atitud positiva hacia el porvenir más bien que a la prudencia.

\section{Introduction}

Les recherches dans le domaine de l'entrepreneuriat et de la PME ont vu l'émergence du concept d'entrepreneurship dont le mérite principal est de considérer l'entrepreneur comme un personnage essentiel au cœur de l'activité entrepreneuriale (Gartner, Shaver, Gatewood et Katz, 1994), et ce, même si le profil des dirigeants d'entreprises est reconnu depuis un certain temps par de nombreux auteurs comme un facteur important quant aux choix stratégiques effectués (Kets de Vries et Miller, 1985), notamment dans les PME (Julien et Marchesnay, 1988). 
Par ailleurs, la percée somme toute récente des sciences de la cognition dans les sciences de gestion suscite une approche renouvelée des organisations, de leurs particularités et des représentations dont elles sont l'objet (Morgan, 1989; Cossette, 1994). Or qui dit représentation dit subjectivité, donc sujet.

Nous proposons de «marier» en quelque sorte les concepts de profil et de représentation en nous intéressant aux valeurs du sujet «clé » qu'est le dirigeant, dans la poursuite des travaux de Bamberger (1980-1987). Ainsi, la question centrale à la base de la recherche effectuée ici peut être formulée comme suit : "Quelles sont les relations qui existent entre les valeurs du dirigeant appréciées en tant que système et le comportement stratégique ?»

Pour répondre à cette question, nous structurerons notre réflexion en trois parties. La première présente le modèle de la recherche menée en s'appuyant sur les aspects théoriques concernés. La deuxième partie explique l'opérationalisation des variables et fixe le cadre méthodologique retenu. La troisième partie énonce et commente les principaux résultats.

\section{Aspects théoriques et modèle de la recherche}

La littérature reconnaît le rôle de l'entrepreneur comme primordial, tant dans la création de l'identité et de la culture de l'entreprise (Larçon et Reitter, 1971 ; Vignon et Zaddem, 1989; Reitter et al., 1991) que dans la prise des décisions stratégiques et dans leur application (Mintzberg, 1990; Julien et Marchesnay, 1988) notamment liées au développement de l'entreprise. En ce qui concerne les PME, la littérature met l'accent sur le fait que, en raison des spécificités de l'organisation et de la direction, une part des atouts de la compétitivité de la PME dépend de la capacité du dirigeant-propriétaire à maîtriser différents niveaux de décision et d'action de l'entreprise (Julien et Marchesnay, 1988).

En revanche, les études ayant mesuré la relation des caractéristiques individuelles du dirigeant ou celles de l'organisation avec les valeurs du dirigeant restent rares ${ }^{1}$.

En deçà du rôle de l'entrepreneur, les valeurs du dirigeant sont considérées, dans la littérature, comme un élément pouvant influencer les comportements organisationnel et stratégique des entreprises (Bamberger et Gabele, 1980; Moss Kanter, 1984; Kets de Vries et Miller, 1985; Miller et Toulouse, 1986; Julien et Marchesnay, 1988; Mahé de Boislandelle, 1988; Pras et Roux, 1990; Blais et Toulouse, 1990).

1. Citons cependant le travail de Stoetzel sur Les valeurs du temps présent, 1983, qui établit des relations entre l'âge, le sexe, l'emploi, la profession, le niveau d'instruction, le revenu, la propriété, d'une part, et les valeurs, d'autre part. 
L'influence reconnue du dirigeant quant au comportement stratégique de la PME, d'une part, la place centrale et fondatrice des valeurs sur la personnalité et les comportements humains, d'autre part, nous invitent à explorer plus avant le système de valeurs du dirigeant à la suite des travaux de Bamberger, en proposant le modèle ci-après :

Figure 1

Modèle de la recherche

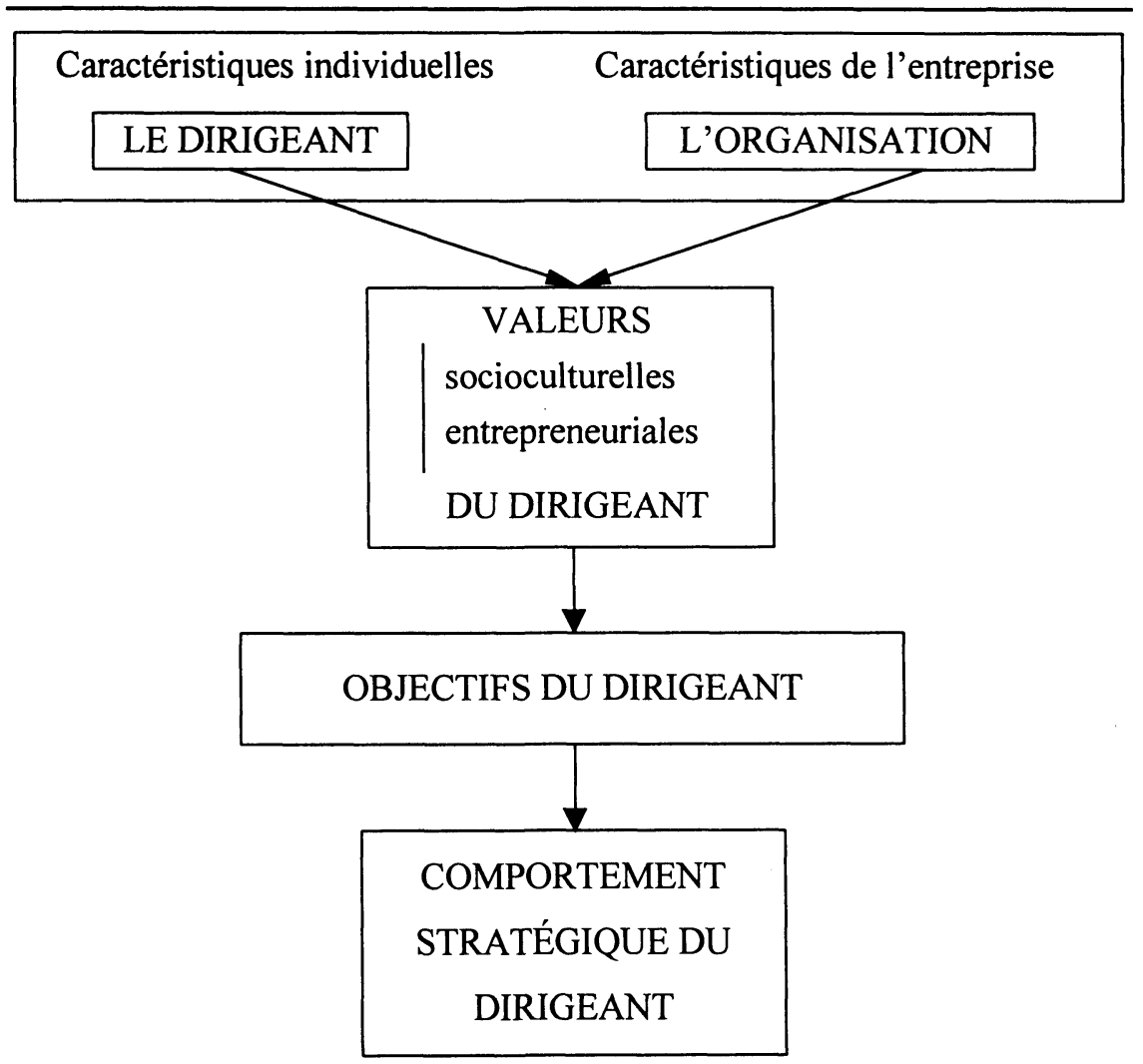

\subsection{Les valeurs du dirigeant}

Dans les sciences humaines, les valeurs comptent parmi les concepts théoriques fondamentaux pour expliquer le comportement des individus et des systèmes sociaux. Elles constituent des représentations mentales, parfois inconscientes, et font partie de la personnalité de l'individu, qu'elles prédisposent à certains 
comportements. La personnalité peut se définir comme l'ensemble relativement stable des caractéristiques d'un individu (Julien et Marchesnay, 1988).

Les valeurs personnelles sont constituées par l'ensemble des principes moraux, des préférences culturelles et des attitudes psychologiques qui structurent les jugements des dirigeants (Strategor, 1991). Ainsi, des structures d'attitudes s'organisent autour de buts extrêmement larges (Newcomb, Turner et Converse, 1970). Alors, les valeurs peuvent être considérées comme l'ultime développement des nombreux processus de sélection et de généralisation qui donnent aux comportements individuels une constante et une organisation à long terme. Rappelons la définition donnée par Milton Rockeach (cité dans Gauthey, Ratiu, Rodgers et Xardel, 1988): «Dire qu'une personne a des valeurs signifie qu'elle a en elle la croyance profonde qu'un mode spécifique de conduite est personnellement ou socialement préférable à d'autres. » Les valeurs sont des standards (normatifs) pour ce qui est considéré comme "bon », «désirable » ou "préférable» et les attitudes sont des valeurs dérivées (Bamberger et Gabele, 1980) ou des expressions reliées à une ou plusieurs valeurs (Gauthey, Ratiu, Rodgers et Xardel, 1988).

L'étude des valeurs aboutit à des classifications diverses en fonction notamment des normes culturelles et collectives, du champ disciplinaire dans lequel s'inscrit l'étude et du référentiel de l'auteur (Kostenbaum, 1989; Newcomb, Turner et Converse, 1970; Allport, Vernon et Lindzey, 1951; Spranger, 1928, cité dans Bamberger et Gabele, 1980). Les différentes fonctions théoriques attribuées aux valeurs sont résumées par Bamberger et Gabele (1980) :

- des stabilisateurs de la personnalité;

- des promoteurs d'adaptation (dans le processus de socialisation, d'internalisation, d'identification, etc.);

- des stabilisateurs de systèmes sociaux ;

- des facteurs dirigeant l'orientation de l'homme.

Dans le modèle proposé, nous distinguons valeurs socioculturelles et valeurs entrepreneuriales. Les premières font référence aux valeurs philosophiques traditionnelles (Stoetzel, 1983), les secondes renvoient à des valeurs pratiques (Allport et al., 1951) dans le champ managérial et stratégique.

\subsection{Les objectifs du dirigeant}

Plusieurs études ont été menées sur les objectifs des entrepreneurs et des entreprises ; elles concernent les grandes, mais aussi les moyennes et petites entreprises. La plupart aboutissent à des typologies. Les objectifs considérés comme discriminants sont souvent la croissance, l'indépendance et la performance économique. L'étude des objectifs peut procéder de la volonté implicite ou explicite de déterminer les 
«ingrédients» de la performance qu'elle soit d'abord économico-financière ou sociale et managériale. Plusieurs typologies issues des recherches en la matière sont synthétisées dans le tableau 1.

\section{TABleau 1}

Typologies des entrepreneurs selon différents auteurs

\begin{tabular}{|c|c|c|c|c|c|}
\hline Auteurs & $\begin{array}{c}\text { Boswell } \\
\text { (1971) }\end{array}$ & $\begin{array}{l}\text { Laufer } \\
\text { (1975) }\end{array}$ & $\begin{array}{l}\text { Lecointre } \\
\text { (1977) }\end{array}$ & $\begin{array}{c}\text { Marchesnay } \\
\text { (1988) }\end{array}$ & $\begin{array}{l}\text { Marmuse } \\
\text { 1992) }\end{array}$ \\
\hline $\begin{array}{l}\text { Typologies } \\
\text { proposées }\end{array}$ & $\begin{array}{l}\text { - conservateurs } \\
\text { - rationnels } \\
\text { - expansionnistes }\end{array}$ & $\begin{array}{l}\text { - entrepreneur } \\
\text { manager et } \\
\text { innovateur } \\
\text { - entrepreneur } \\
\text { propriétaire } \\
\text { - entrepreneur } \\
\text { technicien } \\
\text { - entrepreneur } \\
\text { artisan }\end{array}$ & $\begin{array}{l}\text { - } \begin{array}{l}\text { organisations } \\
\text { familiales }\end{array} \\
\text { autonomes de } \\
\text { production } \\
\text { - entreprises } \\
\text { de survie } \\
\text { - entreprises } \\
\text { à croissance } \\
\text { volontariste }\end{array}$ & $\begin{array}{l}\text { - entrepreneur } \\
\text { «PIC } \\
\text { (pérennité- } \\
\text { indépendance- } \\
\text { croissance } \\
\text { - entrepreneur } \\
\text { «CAP» } \\
\text { (croissance- } \\
\text { autonomie- } \\
\text { pérennité) }\end{array}$ & $\begin{array}{l}\text { - artisan } \\
\text { - entrepreneur } \\
\text { - manager }\end{array}$ \\
\hline
\end{tabular}

La plupart des typologies que nous avons consultées aboutissent à déterminer des profils types pour le dirigeant, profils qui présentent une certaine permanence : le dirigeant recherche plus ou moins la croissance dans une volonté d'expansion, il peut être dans la gestion plus ou moins conservatrice du patrimoine familial dont il a hérité la charge, ou être un manager plus ou moins affirmé.

La littérature se contente, la plupart du temps, de décrire les profils du dirigeant à partir des objectifs poursuivis, au mieux en établissant des typologies, mais sans fournir une explication qui nous semble possible, en considération des travaux de Stoetzel et Bamberger, par l'étude du système de valeurs. Ainsi, le système de valeurs pourrait être considéré comme une variable latente dont les objectifs ne sont que des indicateurs dans un environnement donné.

\subsection{Système de valeurs et comportement stratégique}

Les recherches empiriques concernant les PME établissent de manière de plus en plus fine les relations existant entre le dirigeant, sa personnalité, son profil, son style de direction, sa vision, d'une part, et les comportements stratégiques, organisationnels et la performance de l'entreprise, d'autre part. Le tableau 2 présente succinctement, à titre d'illustration, quelques-uns des travaux de recherche étudiant ces relations. 


\section{TABLEAU 2}

\section{Recherches effectuées dans le champ étudié}

\begin{tabular}{ll}
\hline Auteurs & \multicolumn{1}{c}{ Relations étudiées } \\
\hline Kashefi-Zihagh (1970) & Systèmes de valeurs et efficacité organisationnelle. \\
England (1975) & Systèmes de valeurs et organisation, stratégie, décision, performance. \\
Bamberger et Gabele (1980) & Systèmes de valeurs et comportements stratégiques. \\
Moss Kanter (1984) & Composantes individuelles et structure de l'organisation. \\
Kets de Vries et Miller (1985) & Psychologie du dirigeant et comportement organisationnel. \\
Miller et Toulouse (1986); & Personnalité du dirigeant et comportements stratégiques et \\
Miller, Droge et Toulouse (1988) & organisationnels. \\
Julien et Marchesnay (1988) & Personnalité du dirigeant et processus stratégique et performance. \\
Mahé de Boislandelle (1988) & Caractéristiques individuelles du dirigeant et variables organisationnelles. \\
Frank et al. (1989) & Valeurs du dirigeant et planification. \\
Benoît et Rousseau (1990) & Vision du dirigeant et gestion des ressources humaines. \\
Blais et Toulouse (1990) & Motivations des entrepreneurs et création d'entreprise. \\
Carrière (1990) & Vision du décideur et stratégie. \\
Pras et Roux (1990) & Variables individuelles interculturelles et stratégie de croissance. \\
Lefebvre (1992) & Profil du dirigeant et innovation technologique de l'entreprise. \\
Bayad et Herrmann (1992) & Profil du dirigeant et pratiques de financement. \\
Cossette (1994) & Cartes cognitives et organisation. \\
\hline
\end{tabular}

Les variables mises en relation dans ces travaux nous permettent de discerner trois modes de relations différents :

- le mode classique, qui met en rapport des variables individuelles (caractéristiques objectives, observables et mesurables : âge, nationalité, niveau de formation, expérience, etc.) avec le comportement stratégique ou organisationnel de l'entreprise ;

- le mode psychosociologique, qui met en rapport des variables plus délicates à appréhender concernant la psychologie, la personnalité et la motivation du dirigeant. Ces éléments sont également mis en relation avec le comportement stratégique ou organisationnel de l'entreprise ;

- le mode émergent, enfin, qui met en relation la vision du dirigeant, en essayant par le biais de la cartographie cognitive d'objectiver la singularité individuelle et de mettre au jour les processus décisionnels du dirigeant.

Le système de valeurs du dirigeant de PME, tel qu'il a été défini par Rockeach, peut être appréhendé comme cristallisant les composantes individuelles, psychologiques et cognitives et les processus décisionnels du dirigeant. 
Le comportement stratégique retenu pour l'étude ci-après est celui de la croissance. Ce choix est motivé par plusieurs facteurs :

- la croissance est un point de passage nécessaire pour la majorité des PME ;

- la croissance est également un choix stratégique issu d'une décision importante de gestion pour constituer un avantage concurrentiel déterminant (Marmuse, 1992; Strategor, 1991);

- la croissance devient un axe crucial au regard de l'évolution de l'environnement des entreprises.

La faible importance des études effectuées sur les relations entre systèmes de valeurs et comportements stratégiques, et leur absence, à notre connaissance, quant au comportement de croissance, toujours en relation avec le système de valeurs du dirigeant, nous invitent à réaliser une étude :

- qui décrit une population donnée suivant ses systèmes de valeurs,

- qui apprécie, ensuite, les relations entre systèmes de valeurs et le comportement stratégique qu'est la croissance.

\section{Aspects méthodologiques}

La problématique et le modèle énoncés permettent d'avancer les hypothèses ci-après qui seront vérifiées empiriquement au moyen d'un questionnaire utilisant la technique des indicateurs et administré dans un secteur d'activité donné afin de réduire les influences sectorielles.

\subsection{Les hypothèses}

Les hypothèses de la recherche reprennent en partie les hypothèses du grand projet initial de Bamberger de manière moins ambitieuse, mais plus spécialisée (un seul secteur d'activité, une nationalité, un créneau d'effectif, un type de comportement stratégique). L'enjeu est de dépasser le stade descriptif réalisé par Stratos (1987) et Bamberger, Gueye, Moussayir et Robic (1986), en attribuant du sens au système de valeurs et en amorçant une explication de la relation entre valeurs et croissance.

1. «Les valeurs sont structurées en un système cohérent permettant de dresser le profil du dirigeant. » Cette première hypothèse permet d'apprécier les valeurs en tant que système socioculturel à partir des mesures fournies par des indicateurs.

2. «Les valeurs des dirigeants définissant la croissance comme un objectif prioritaire diffèrent de celles des autres dirigeants. » Dans la deuxième hypothèse, suivre un objectif de croissance prioritaire devient un premier 
critère de différenciation, au regard du système de valeurs, au sein de la population étudiée.

\subsection{Les variables}

À la lumière des études déjà réalisées, cinq familles de variables sont constituées :

- Les caractéristiques individuelles du dirigeant : âge, statut marital, nombre d'enfants, niveau de formation, expérience dans la fonction, degré de participation au capital (Pras et Roux, 1990; Bamberger, Gueye, Moussayir et Robic 1986; Stratos, 1987).

- Les caractéristiques de l'organisation : nombre d'années d'existence, phase de développement, chiffre d'affaires, structuration éventuelle en filiale, recours à la sous-traitance, taux de croissance annuel et celui espéré dans cinq ans (Pras et Roux, 1990; Bamberger, Gueye, Moussayir et Robic, 1986).

- Les valeurs et attitudes socioculturelles : importance accordée aux valeurs religieuses, politiques, familiales, sentiment de réussite psychologique, professionnelle, relationnelle (Stoetzel, 1983).

- Les valeurs entrepreneuriales : degré d'acceptation du risque, importance accordée à la croissance, au profit, à la sécurité, aux investissements, aux placements, aux changements technologiques, volonté d'indépendance financière, d'indépendance à l'égard des salariés, des clients et des fournisseurs, sens que revêt la possession d'un patrimoine, sens accordé à la création et au développement d'une entreprise.

- Les objectifs poursuivis par le dirigeant : rentabilité et productivité, maximisation du bénéfice, croissance, leadership dans la branche, stabilité, efficacité technique, bien-être des salariés (Bayad et Paradas, 1995; Lorrain et Dussault, 1988; Mahé de Boislandelle, 1988 ; Bamberger, Gueye, Moussayir et Robic, 1986 ; Lecointre, 1977 ; Laufer ; 1975 ; England et Lee, 1973 ; Boswel, 1971).

Les objectifs sont appréciés directement par des questions relatives à l'importance de certains d'entre eux, mais aussi, indirectement, par le biais des valeurs considérées comme des prémisses décisionnelles influençant le choix des objectifs. La plupart des variables sont de nature qualitative.

Pour pallier la difficulté inhérente à l'observation et à la mesure des valeurs, différentes techniques ont été mises au point, dont chacune offre un intérêt relatif en fonction de la nature de l'étude et de son objet. Bamberger et Gabele (1980) citent les techniques suivantes auxquelles il conviendrait d'ajouter les tests psychologiques : 
- $\quad$ observation du comportement ;

- procédés verbaux faiblement structurés;

- techniques sur la base des sémantiques différentielles ;

- méthode de classement hiérarchique ;

- $\quad$ techniques sur la base d'indicateurs.

C'est ce dernier procédé que nous utilisons au moyen d'un questionnaire. Les valeurs sont appréhendées au moyen d'indicateurs, manifestations mesurables et observables des composantes du concept (Quivy et Van Campenhoudt, 1988). Une partie des indicateurs qui seront utilisés dans le cadre de la recherche sont des appréciations subjectives exprimant des perceptions ou des opinions. Notons que ces indicateurs portent sur des appréciations moins impliquantes que si les valeurs étaient appréhendées directement. Les indicateurs permettent ainsi une réduction du biais inhérent à la subjectivité.

Le secteur choisi pour l'investigation est celui de la plasturgie : la plasturgie est une industrie relativement jeune qui connaît une croissance persistante malgré le ralentissement de $1993^{2}$. Ce secteur compte près de 4400 entreprises en France (cinquième pays producteur) et occupe près de 146600 salariés, répartis à $80 \%$ dans des PME et des $\mathrm{PMI}^{3}$.

La taille retenue pour la sélection des entreprises est de 100 à 200 salariés ; elle paraît suffisante pour permettre une explicitation de la stratégie en matière de croissance, tout en laissant une place importante à l'influence des valeurs personnelles du dirigeant. La sélection a été opérée à partir de la base de données du Kompass en 1993, ce qui a permis le recueil de 500 entreprises auxquelles a été adressé un questionnaire par voie postale, avec un taux de réponse de 8,5\% (mai-juin 93).

Le questionnaire comporte trois grandes parties : le dirigeant, ses valeurs; l'entreprise, ses objectifs ; les éléments de contexte. En ce qui concerne l'investigation des valeurs, nous avons étudié le travail du European Value Systems Study Group $^{4}$. Cette équipe de recherche a réalisé une enquête sur les valeurs en Europe occidentale (Stoetzel, 1983).

2. Les chiffres communiqués par la Fédération française de la plasturgie donnent un taux de croissance moyen pour 1990-1995 de 2,64\% pour les entreprises de plus de 20 salariés. Ce chiffre moyen doit être pondéré par le net ralentissement enregistré en 1993 $(-7 \%)$, puis la reprise de 1994 et $1995(+7 \%)$.

3. «Quand la plasturgie met le turbo », Objectifformation Lorraine, avril 1994, p. 22-23.

4. Groupe européen d'étude sur les systèmes de valeurs ayant son siège à Amsterdam.

5. Enquête européenne par sondage effectuée dans neuf pays en 1981, au cours de laquelle les réponses de 12463 personnes ont été recueillies dont 1199 en France. 
TABLEAU 3

Opérationalisation des variables « valeurs »

VARIABLES OPÉRATIONALISATION

\section{SOCIOCULTURELLES}

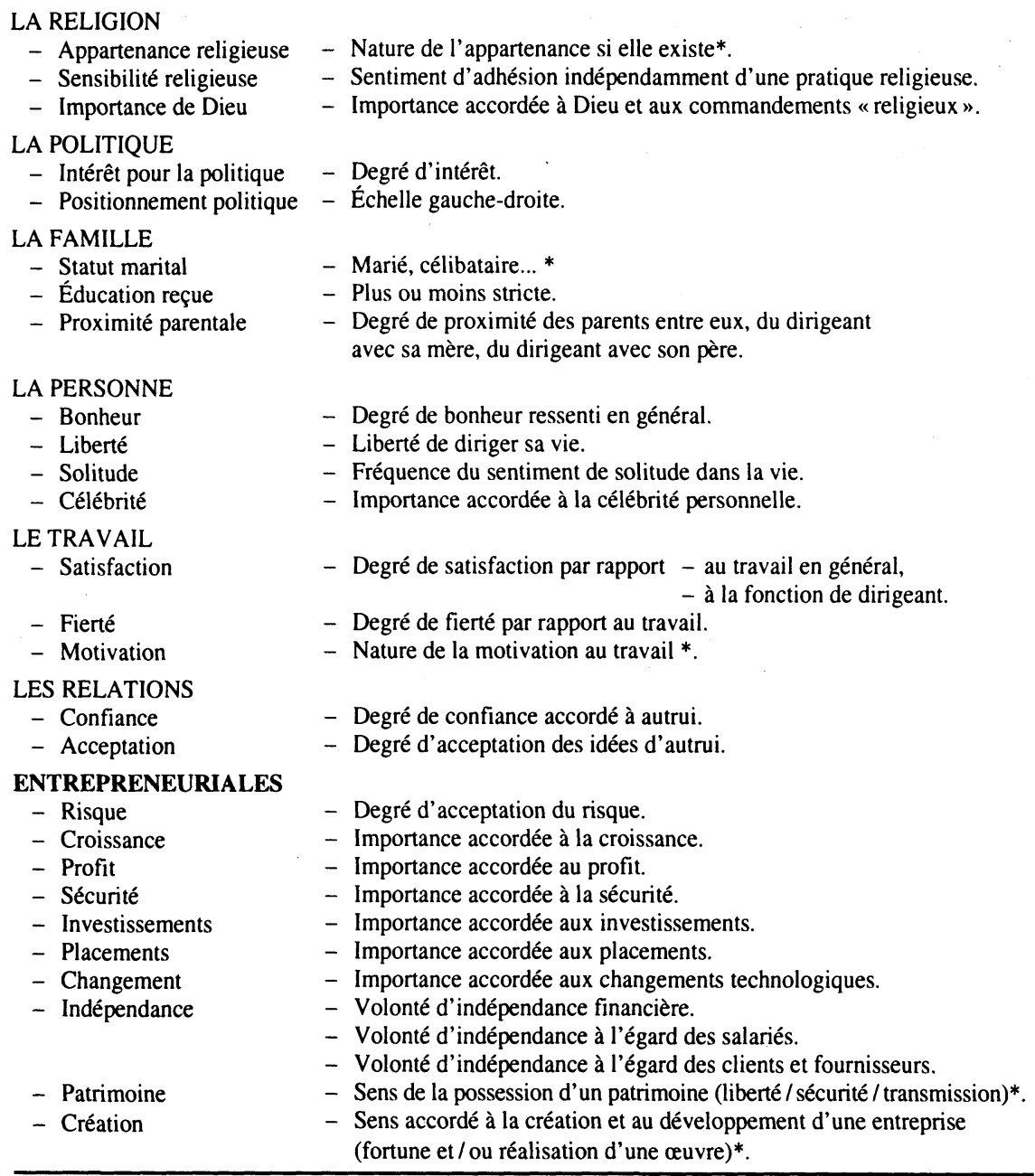

Note: L'ensemble des indicateurs sont appréciés par des échelles d'attitudes de Lickert en six points et ceux auxquels on a apposé un astérisque reposent sur des questions à choix multiples.

Les 70 questions du questionnaire se répartissent en une majorité de questions fermées à plusieurs modalités, organisées autour de quelques questions ouvertes permettant un enrichissement des réponses sur les objectifs poursuivis. Différentes 
techniques d'analyses statistiques des données sont utilisées. L'analyse descriptive permet de repérer les valeurs, les objectifs et les stratégies poursuivis par les dirigeants. Des tris à plat et des tris croisés sont effectués pour chaque variable (analyse unidimensionnelle). Les analyses factorielles des correspondances multiples effectuées sur les variables de valeurs permettent de déterminer les axes principaux (analyse multidimensionnelle). Le traitement des données a été effectué à l'aide des logiciels SYSTAT et STATITCF.

\section{Résultats et commentaires}

L'ensemble des résultats présentés ci-après repose sur le traitement des réponses collectées (taux de réponse 8,5\%). Dans une première partie, nous présentons le profil socioculturel, puis entrepreneurial des dirigeants. Dans une deuxième partie, après avoir décrit les objectifs poursuivis par les entreprises répondantes, nous analysons la relation des valeurs avec l'objectif de croissance. Pour finir, nous prendrons en compte les variables de contexte. En préalable, nous proposons une rapide description de l'échantillon des répondants.

\section{Le dirigeant}

Sur 10 dirigeants, 6 ont entre 40 et 50 ans (âge moyen 47 ans), 8 ont un niveau de formation supérieure, 9 sont mariés et de sexe masculin. Presque tous ont des enfants, souvent 2 et plus. Une fois sur deux, le dirigeant est salarié. Quand ce n'est pas le cas, il a créé ou racheté l'entreprise ( 3 sur 10 ) ou l'a acquise par succession ( 2 sur 10 ). Il détient une partie du capital 1 fois sur 2 , et 2 fois sur 3, il occupe la fonction de dirigeant depuis moins de 10 ans avec une expérience moyenne du secteur de 15 années. L'échantillon est homogène en ce qui concerne le statut familial et le sexe. En revanche, seule une moitié des dirigeants détient une partie plus ou moins importante du capital.

\section{L'entreprise}

Jeune (une fois sur deux, elle a moins de 20 ans), bénéficiaire ( 3 sur 4 ), optimiste (taux de croissance espéré positif), relativement dépendante ( 1 sur 2 est structurée en filiale), en phase de maintien ou de développement ( 9 sur 10), innovante (plus de 7 produits nouveaux en moyenne dans les cinq dernières années).

L'échantillon est ainsi homogène en ce qui concerne la phase de développement de l'entreprise et sa rentabilité; en revanche, une hétérogénéité majeure apparaît en ce qui concerne la structuration en filiale. 


\subsection{Profils types des valeurs du dirigeant}

Les profils des valeurs des dirigeants interrogés proviennent des résultats issus des analyses unidimensionnelles et multidimensionnelles. Ces valeurs peuvent être appréciées sous deux angles : les valeurs à orientation socioculturelle et les valeurs à orientation entrepreneuriale.

\subsubsection{Profil socioculturel: tradition et New Age}

Les résultats principaux de l'analyse unidimensionnelle, présentés dans le tableau 4, permettent une description de valeurs fortes et structurées. L'intérêt des dirigeants pour la politique semble manifeste. La dimension spirituelle existe, mais sans pratique religieuse. La plupart des dirigeants ont bénéficié d'une éducation classique et protégée sur le plan affectif et vivent à présent des relations familiales harmonieuses. La position philosophique dominante se caractérise par un sentiment de libre-arbitre avec un accès certain au bonheur, même s'il reste difficile de faire totalement confiance à autrui. Le sentiment de satisfaction dans le travail domine avec la motivation et la fierté ad hoc.

TABLEAU 4

Profil des valeurs sociologiques des dirigeants des moyennes entreprises

\begin{tabular}{llc}
\hline Valeurs sociologiques & \multicolumn{1}{c}{ Profil des dirigeants } & Pourcentage \\
\hline Liées à la politique & - intéressés par la politique & $91 \%$ \\
& - au centre vers la droite & $82 \%$ \\
Liées à la religion & - catholiques & $71 \%$ \\
& - non religieux & $82 \%$ \\
Liées à l'éducation & - sensibles à Dieu & $62 \%$ \\
& - proximité des parents entre eux & $85 \%$ \\
& - proximité avec la mère & $79 \%$ \\
Liées au travail & - proximité avec le père & $70 \%$ \\
& - éducation stricte & $79 \%$ \\
& - tout à fait satisfaits de leur travail & $79 \%$ \\
& - relativement fiers de leur travail & $77 \%$ \\
& - motivés par l'intérêt du travail & $73 \%$ \\
& - motivés par les responsabilités & $68 \%$ \\
Liées à la personne & - motivés par la prise d'initiatives & $56 \%$ \\
& - motivés par l'ambiance de travail & $53 \%$ \\
Liées à autrui & - heureux & $97 \%$ \\
& - libres de diriger leur vie & $88 \%$ \\
& - relativement intéressés par la célébrité & $53 \%$ \\
& - relativement confiants & $56 \%$ \\
\hline
\end{tabular}


L'analyse factorielle des correspondances permet la mise en relief des valeurs déterminantes (tableau 5). Les valeurs religieuses semblent expliquer le mieux le premier axe. Le deuxième axe est déterminé par des variables concernant les valeurs relatives à la famille et à la personne. Le troisième axe semble issu de variables psychologiques et relationnelles. Les valeurs politiques n'apparaissent pas, contrairement à ce qu'on a observé dans les résultats précédents.

TABLEAU 5

Pourcentages de l'inertie calculés à partir du tableau de contingence

\begin{tabular}{|c|c|c|c|c|c|}
\hline \multirow[t]{2}{*}{$\begin{array}{c}\text { Axe } \\
\text { factoriel }\end{array}$} & \multicolumn{2}{|c|}{$\begin{array}{l}\text { Pourcentage } \\
\text { d'inertie }\end{array}$} & \multirow[t]{2}{*}{ Valeurs explicatives des axes } & \multirow{2}{*}{$\begin{array}{l}\text { Contributions } \\
\text { à l'inertie } \\
(\%)\end{array}$} & \multirow[t]{2}{*}{$\begin{array}{l}\text { Coor- } \\
\text { données }\end{array}$} \\
\hline & simple & cumulé & & & \\
\hline F1 & 35,6 & 35,6 & $\begin{array}{l}\text { Appartenance à une religion } \\
\text { Importance de Dieu dans la vie } \\
\text { Croyance en Dieu }\end{array}$ & $\begin{array}{c}57,9 \\
23,4 \\
6,6\end{array}$ & $\begin{array}{l}-0,859 \\
+0,450 \\
+0,538\end{array}$ \\
\hline F2 & 10,7 & 46,3 & $\begin{array}{l}\text { Importance accordée à la célébrité } \\
\text { Proximité du dirigeant avec son père } \\
\text { Proximité entre les parents du dirigeant }\end{array}$ & $\begin{array}{l}32,2 \\
19,9 \\
18,3\end{array}$ & $\begin{array}{l}+0,308 \\
-0,279 \\
-0,288\end{array}$ \\
\hline F3 & 9,7 & 56,0 & $\begin{array}{l}\text { Bonheur personnel } \\
\text { Confiance des jeunes en leurs aînés } \\
\text { Bonheur en famille } \\
\text { Anxiété }\end{array}$ & $\begin{array}{l}19,1 \\
10,9 \\
9,1 \\
8,0\end{array}$ & $\begin{array}{l}+0,303 \\
-0,156 \\
+0,242 \\
-0,157\end{array}$ \\
\hline
\end{tabular}

À première vue, le profil du dirigeant semble traditionnel. En effet, l'importance des valeurs religieuses et des valeurs familiales ressort dans les deux analyses : catholiques par la culture plus que par choix, avec une sensibilité à Dieu, mais sans allégeance ni pratique religieuses. L'importance accordée aux valeurs familiales traditionnelles peut provenir de l'éducation reçue (stricte, union des parents) et s'affiche dans le mode d'existence actuelle: famille structurée avec stabilité du couple marié et la présence de deux enfants ou plus. Le positionnement politique ne revêt qu'une importance relative, mais sans surprise : position centre droite ; attitudes politiques et religieuses souvent associées (Stoetzel, 1983).

La dimension la plus novatrice dans ce profil que nous avons étiqueté comme traditionnel au regard des résultats antérieurs obtenus par Stoetzel auprès de la population française douze ans plus tôt semble être marquée par un positionnement libéral, un climat de bonheur, assis sur le sentiment du libre arbitre. On peut supposer un recul des valeurs collectives (les institutions perdent de leur crédit : pas d'engagement politique, ni de pratique religieuse) au profit de valeurs «individualisées », telles que le développement personnel dans le cadre privé, l'accomplissement par le travail et la fonction, et la sensibilité spirituelle sans allégeance institutionnelle. Recul des institutions (mais pas de leur objet) et augmentation du sentiment de 
responsabilité individuelle. S'agit-il du nouveau paradigme vulgarisé sous le vocable «New Age»? (Ferguson, 1981). Est-ce lié au fait que le dirigeant a le sentiment de prendre son destin en mains et qu'il en est heureux ? Un croisement des variables valeurs entre elles permettrait de répondre notamment à cette question relative à une problématique élargie par rapport à celle de la recherche énoncée.

\subsubsection{Profil entrepreneurial: réalisme}

Les principaux résultats de l'analyse unidimensionnelle sont présentés dans le tableau 6. Aucun dirigeant ne se situe en position d'évitement du risque. Profit, sécurité et croissance semblent importants. On considère qu'il vaut mieux investir et effectuer des changements technologiques que de réaliser des placements. L'indépendance financière est essentielle ainsi que l'indépendance à l'égard des clients et des fournisseurs. La possession d'un patrimoine représente avant tout la possibilité d'être libre et en sécurité, sans grand désir de faire fortune mais avec le souhait de réaliser son œuvre propre.

\section{TABLEAU 6 \\ Profil des valeurs entrepreneuriales des dirigeants des moyennes entreprises}

\begin{tabular}{llc}
\hline Valeurs entrepreneuriales & \multicolumn{1}{c}{ Profil des dirigeants } & Pourcentage \\
\hline Le risque & - acceptent le risque. & $85 \%$ \\
La croissance & - lui accordent beaucoup d'importance. & $67 \%$ \\
Le profit & - lui accordent beaucoup d'importance. & $76 \%$ \\
La sécurité & - lui accordent beaucoup d'importance. & $68 \%$ \\
Les investissements & - leur accordent beaucoup d'importance. & $97 \%$ \\
Les placements & - leur accordent peu d'importance. & $74 \%$ \\
Le changement technologique & - lui accordent beaucoup d'importance. & $91 \%$ \\
L'indépendance financière & - lui accordent beaucoup d'importance. & $85 \%$ \\
L'indépendance à l'égard des salariés & - lui accordent une importance moyenne. & $55 \%$ \\
L'indépendance à l'égard des clients & - lui accordent de l'importance. & $73 \%$ \\
et fournisseurs & & \\
Le patrimoine & - considéré comme un instrument & $52 \%$ \\
$\begin{array}{l}\text { La création et le développement } \\
\text { de l'entreprise }\end{array}$ & de liberté. & $62 \%$ \\
\hline
\end{tabular}

L'analyse factorielle des correspondances permet une représentation synthétique des valeurs entrepreneuriales déterminantes des dirigeants présentées ci-dessous. Dans l'explication du premier axe, l'importance accordée au fait de réaliser des placements contribue négativement ainsi que l'importance accordée à 
l'indépendance à l'égard des salariés. Le deuxième axe est expliqué par l'importance accordée à l'indépendance à l'égard des clients et fournisseurs, ensuite de nouveau l'indépendance à l'égard des salariés. Enfin, le troisième axe est expliqué négativement par la création et le développement de l'entreprise et positivement par l'intervention de l'État dans l'économie.

TABLEAU 7

Pourcentages de l'inertie calculés à partir du tableau de contingence

\begin{tabular}{|c|c|c|c|c|c|}
\hline \multirow[t]{2}{*}{$\begin{array}{c}\text { Axe } \\
\text { factoriel }\end{array}$} & \multicolumn{2}{|c|}{$\begin{array}{l}\text { Pourcentage } \\
\text { d'inertie }\end{array}$} & \multirow[t]{2}{*}{ Valeurs explicatives des axes } & \multirow{2}{*}{$\begin{array}{c}\text { Contributions } \\
\text { à l'inertie } \\
(\%)\end{array}$} & \multirow[t]{2}{*}{$\begin{array}{l}\text { Coor- } \\
\text { données }\end{array}$} \\
\hline & simple & cumulé & & & \\
\hline \multirow[t]{2}{*}{ Fl } & 18,7 & 18,7 & Placements & 50,3 & $-0,397$ \\
\hline & & & Indépendance à l'égard des salariés & 14,1 & $-0,194$ \\
\hline \multirow[t]{2}{*}{ F2 } & 15,2 & 33,9 & $\begin{array}{l}\text { Indépendance à l'égard des clients } \\
\text { et fournisseurs }\end{array}$ & 26,9 & $+0,218$ \\
\hline & & & Indépendance à l'égard des salariés & 13,5 & $+0,171$ \\
\hline \multirow[t]{2}{*}{ F3 } & 11,7 & 45,6 & Création et développement de l'entreprise & 33,7 & $-0,284$ \\
\hline & & & Intervention de l'État dans l'économie & 19,0 & $+0,167$ \\
\hline
\end{tabular}

Sur le plan entrepreneurial, les chiffres manifestent le réalisme des dirigeants qui intègrent un ensemble d'enjeux parfois contradictoires. Croissance et sécurité sont également considérées comme importantes dans une relation plus dialectique que conflictuelle. Le risque est admis comme une donnée effective dans l'entrepreneuriat sans pour autant nier l'importance de la sécurité. Enfin, si la satisfaction par rapport au travail est de règle (Bamberger, Gueye, Moussayir et Robic, 1986), la nature des motivations varie (Burke, 1991). Rappelons qu'une position à statut élevé, avec les rôles qu'elle engendre, offre des satisfactions (Newcomb, Turner et Converse, 1970). Les dirigeants témoignent avant tout d'une volonté d'indépendance (Julien et Marchesnay, 1988) assortie de l'importance accordée au profit (Bamberger, Gueye, Moussayir et Robic, 1986) tout en souhaitant réaliser une œuvre (Amiot, 1991 ; Van Loye, 1991). L'investissement productif peut être interprété comme étant un moyen «plus moral» d'accès au profit que le placement.

\section{Le dirigeant pilote}

La synthèse des deux approches, socioculturelle et entrepreneuriale, peut être symbolisée par le concept de pilotage. Le dirigeant semble avoir acquis le bonheur grâce au sentiment de piloter son existence personnelle. De même, en pilote averti, il est conscient des enjeux de développement pour l'entreprise, en intégrant les contraintes inhérentes à la sécurité et en maintenant son désir d'indépendance. 


\subsection{La relation entre valeurs et croissance}

En préambule, nous dressons le profil des objectifs déclarés poursuivis par les dirigeants répondants. Ensuite, nous décrivons les relations pouvant exister entre valeurs, d'une part, et objectifs de croissance, d'autre part.

\subsubsection{Profil des objectifs poursuivis par les dirigeants}

Il est, bien sûr, légitime pour une entreprise de satisfaire aux objectifs de rentabilité et de productivité ; notons cependant que la maximisation du bénéfice en soi n'est que très peu citée, fait à rapprocher de l'intérêt très relatif pour les placements (voir tableau 7 et 6). Le profit pour le profit apparaît comme suspect. Ces résultats confirment en partie ceux de Bamberger, Gueye, Moussayir et Robic (1986) dans lesquels rentabilité, productivité, bénéfices, part de marché et croissance apparaissent dans cet ordre d'importance.

TABlEAU 8

Objectifs déclarés prioritaires

\begin{tabular}{lc}
\hline Objectif & Pourcentage \\
\hline Rentabilité et productivité & $88 \%$ \\
Bien-être des salariés & $62 \%$ \\
Leadership dans la branche & $44 \%$ \\
Efficacité technique & $41 \%$ \\
Croissance & $\mathbf{3 8 \%}$ \\
Stabilité & $8,8 \%$ \\
Maximisation du bénéfice & $5,9 \%$ \\
\hline
\end{tabular}

Un résultat moins attendu est la nette préoccupation du dirigeant pour le bien-être des salariés, déjà identifié cependant comme plus important dans les très petites entreprises (England et Lee, 1973). L'objectif croissance est le plus souvent associé à celui de rentabilité et de productivité, ainsi qu'au bien-être des salariés. En effet, en situation de croissance réussie, il paraît légitime d'en partager les fruits (Amiot, 1991). La croissance, en tant que telle, n'apparaît pas comme un objectif prioritaire pour la majorité, bien qu'elle soit considérée le plus souvent comme importante (tableau 6). Est-ce à dire que la croissance est plus un moyen au service d'un objectif, qu'un objectif en soi6 ?

6. La croissance prend un sens différent dans les entreprises de plus grande taille (England et Lee, 1973). 


\subsubsection{La relation entre valeurs et objectifs de croissance}

Les résultats ci-après sont issus de l'analyse bidimensionnelle. Rappelons que près de 4 dirigeants sur 10 considèrent la croissance comme un de leurs objectifs prioritaires. Pour cette analyse, nous utilisons différents tableaux de contingence qui mettent en relation les catégories de valeurs avec les deux groupes de dirigeants. Ces groupes se différencient selon qu'ils ont choisi ou non de faire de la croissance un objectif prioritaire.

Les relations que nous présentons ici sont à considérer comme des indications provisoires, des tendances à confirmer. En effet, le test d'indépendance des variables (khi carré) n'est pas statistiquement significatif au seuil de signification de $5 \%$.

TABLEAU 9

Profil des valeurs des dirigeants relatif à l'objectif de croissance

\begin{tabular}{|c|c|c|}
\hline Types de valeurs & $\begin{array}{c}\text { Dirigeants suivant } \\
\text { l'objectif croissance (C) }\end{array}$ & $\begin{array}{l}\text { Dirigeants ne suivant pas } \\
\text { l'objectif croissance (NC) }\end{array}$ \\
\hline \multicolumn{3}{|c|}{ Valeurs socioculturelles } \\
\hline Liées à la politique & $\begin{array}{l}\text { - relativement moins intéressés } \\
\text { par la politique et plus vers la } \\
\text { droite. }\end{array}$ & $\begin{array}{l}\text { - plus intéressés par la } \\
\text { politique et plus vers le } \\
\text { centre. }\end{array}$ \\
\hline Liées à la religion & $\begin{array}{l}\text { - plutôt non religieux ; moins } \\
\text { grande importance de Dieu } \\
\text { dans leur vie. }\end{array}$ & $\begin{array}{l}\text { - plutôt religieux ; plus } \\
\text { grande importance } \\
\text { de Dieu dans leur vie. }\end{array}$ \\
\hline Liées à la famille & $\begin{array}{l}\text { - proximité entre les parents moins } \\
\text { forte ; parents moins stricts ; plus } \\
\text { détendus et heureux quand ils } \\
\text { sont à la maison. }\end{array}$ & $\begin{array}{l}\text { - proximité entre les parents } \\
\text { plus forte ; parents plus } \\
\text { stricts ; moins détendus et } \\
\text { heureux quand ils } \\
\text { sont à la maison. }\end{array}$ \\
\hline Liées au travail & $\begin{array}{l}\text { - plus motivés par la possibilité } \\
\text { de prendre des initiatives ; } \\
\text { préfèrent suivre des instructions } \\
\text { après avoir été convaincus. }\end{array}$ & $\begin{array}{l}\text { - plus motivés par l'intérêt } \\
\text { du travail ; logique de } \\
\text { soumission légitime } \\
\text { à l'autorité. }\end{array}$ \\
\hline $\begin{array}{l}\text { Liées à la personne } \\
\text { (au soi) }\end{array}$ & $\begin{array}{l}\text { - plus heureux, plus libres, } \\
\text { moins seuls. }\end{array}$ & $\begin{array}{l}\text { - relativement moins } \\
\text { heureux, moins libres } \\
\text { et plus seuls. }\end{array}$ \\
\hline Liées à autrui & - font plus confiance à autrui. & $\begin{array}{l}\text { - font moins confiance à } \\
\text { autrui. }\end{array}$ \\
\hline
\end{tabular}

Valeurs

\section{entrepreneuriales}

La croissance, le profit, - accordent plus d'importance la sécurité

Les placements à la croissance et moins au profit et à la sécurité.
- accordent moins d'importance aux placements.

- accordent plus d'importance au profit, à la sécurité et moins à la croissance.

- accordent plus d'importance aux placements. politique et plus vers le

plutôt religieux ; plus grande importance proximité entre les parents orte ; parents plus heureux quand ils plus motivés par l'intérêt a relativement moins heureux, moins libres font moins confiance à 
Des différences peuvent être relevées entre les deux groupes de dirigeants ; mais elles doivent être considérées comme relatives par rapport aux résultats précédents.

En ce qui concerne les variables entrepreneuriales, ces différences portent sur l'importance accordée à la croissance, au profit et à la sécurité. En effet, on retrouve l'opposition attendue entre croissance et sécurité, la recherche de profit et la réalisation de placements étant liées à la sécurité. En fait, les divergences se manifestent davantage dans les valeurs socioculturelles. Le dirigeant qui choisit la croissance se caractérise par un profil de valeurs moins traditionnelles sur les plans politique, religieux et éducatif ; il présente un épanouissement psychologique plus marqué dans le cadre familial actuel, manifeste davantage de confiance dans ses relations avec les autres et se sent libre et heureux. Souvent avec une formation de niveau secondaire, il est jeune et tire sa détermination davantage de sa personnalité que de son expérience. Quant au dirigeant qui n'opte pas pour la croissance a priori, il présente un profil qui renforce l'aspect traditionnel dégagé par l'analyse de la première hypothèse : attrait plus important pour la politique et la religion, logique de soumission légitime à l'autorité. En revanche, il s'éloigne du profil type d'épanouissement psychologique : moindre sentiment de liberté, confiance plus restreinte en autrui, sentiment de solitude accru.

Pour interpréter, tout en restant très prudent, les résultats obtenus par la comparaison des profils de valeurs des dirigeants en fonction du choix affirmé pour la croissance de leur entreprise, il est possible de se référer aux thèses de la psychologie des profondeurs ou psychologie d'inspiration analytique. Ce courant considère la réalité psychique comme élément moteur dans le comportement et les actions de l'individu, ce dernier n'étant pas réductible à ce que l'on peut en observer extérieurement (Chanlat, 1990). Ainsi, le dirigeant, ayant besoin de sécurité dans sa réalité intérieure psychique, peut posséder un système de valeurs où les cadres structurés sont nécessaires, qu'ils soient de nature politique, religieuse ou familiale. En revanche, le dirigeant serait centré sur la croissance de son entreprise, parce qu'il vivrait lui-même une dynamique psychique où la sécurité fondamentale est acquise. Ce qui permet l'initiative, la confiance en autrui, la capacité à se détendre, en réduisant le besoin des cadres structurés. Maslow (1972) souligne le conflit fondamental ancré en chaque être entre les forces défensives (sécurité) et les pulsions de développement (croissance).

Dans l'opposition dialectique individu et collectif, la croissance, selon la thèse psychodynamique, s'enracine davantage dans le premier plan, celui de l'individu, mais en permettant de servir le second, celui de la société, par l'intermédiaire de l'entreprise.

Dans cette optique apparaît dans le tableau 10 un résultat intéressant : il s'agit de la place relative de la prise d'initiatives quant aux motivations concernant le travail. Le groupe «croissance» cite la prise d'initiatives en premier. En revanche, dans l'autre groupe, celle-ci ne se place qu'en cinquième position. Il n'apparaît pas de différence significative quant à l'attitude face à l'indépendance parfois appréhendée comme opposée à la croissance (Julien et Marschenay, 1988). 
TABLEAU 10

Classement des motivations au travail

\begin{tabular}{cll}
\hline $\begin{array}{c}\text { Ordre de } \\
\text { classement }\end{array}$ & $\begin{array}{l}\text { Dirigeants suivant l'objectif } \\
\text { croissance }(\mathbf{C})\end{array}$ & $\begin{array}{l}\text { Dirigeants ne suivant pas } \\
\text { l'objectif croissance (NC) }\end{array}$ \\
\hline $\mathbf{1}$ & Prendre des initiatives & L'intérêt du travail \\
$\mathbf{2}$ & Les responsabilités & Les responsabilités \\
$\mathbf{3}$ & L'intérêt du travail & Bien gagner sa vie \\
$\mathbf{4}$ & Une bonne ambiance de travail & Une bonne ambiance de travail \\
$\mathbf{5}$ & Bien gagner sa vie & Prendre des initiatives \\
\hline
\end{tabular}

Si nous essayons maintenant d'effectuer une lecture des résultats à la lumière des typologies référencées dans la première partie (tableau 1), nous pouvons caractériser le dirigeant déclarant suivre un objectif de croissance :

- comme se rapprochant du type d'entrepreneurs "expansionnistes" (Boswell, 1971) qui recherchent volontairement la croissance, tout en pouvant avoir une attitude différente quant à l'autonomie de l'entreprise ;

- comme appartenant à une entreprise à croissance volontariste (Lecointre, 1977), même si cette typologie porte sur les petites entreprises familiales;

- comme appartenant le plus souvent à la catégorie « entrepreneur, manager, innovateur », ou sinon à celle «entrepreneur propriétaire » (Laufer, 1975). Le premier type favorise la croissance, sa motivation principale étant l'autoréalisation. Le second recherche également la croissance, mais à condition de ne pas remettre en cause son indépendance financière ;

- comme appartenant à la catégorie de l'entrepreneur CAP (croissance, autonomie, pérennité) [Marchesnay, 1988]. Ce type d'entrepreneur, au tempérament opportuniste, met à profit les turbulences de l'environnement pour réaliser des affaires rentables sans que la recherche du profit en tant que telle soit motrice; c'est davantage l'attrait du jeu ou le sentiment d'accomplissement qui peut être décelé ;

- comme ayant dépassé le stade "artisan ", pour accéder au stade «entrepreneur » plutôt que «manager» (Marmuse, 1992). En effet, l' «entrepreneur » gère à partir des objectifs qui sont les siens, cherche à innover, sans craindre la prise de risque.

À ce stade, le dirigeant centré sur l'objectif de croissance semble présenter un certain nombre de caractéristiques tant sur le plan psychologique que managérial. Bénéficiant d'une sécurité personnelle, d'un sentiment de libre arbitre, il peut projeter son désir de développement au sens de Maslow dans sa fonction de dirigeant et suivre un objectif de croissance. Cependant, l'étude menée ne saurait s'abstenir de la prise en compte de variables issues de l'environnement ou du contexte, variables que nous allons considérer à présent. 


\subsection{La prise en compte des autres variables}

Il importe également d'apprécier les relations pouvant exister entre valeurs et objectifs, d'une part, et les variables, relatives au dirigeant, ou relatives à l'entreprise, d'autre part. Rappelons que les relations présentées ici sont à considérer comme des indications provisoires, des tendances à confirmer. En effet, le test d'indépendance des variables (khi carré) n'est pas statistiquement significatif au seuil de signification de $10 \%$ pour la majeure partie des résultats obtenus.

\section{Âge du dirigeant}

Le tableau ci-dessous présente les relations, les plus probables, entre l'âge des dirigeants répondants et les variables relatives aux valeurs et aux objectifs. Trois classes d'âge ont été retenues.

La période 41-50 ans apparaît comme une phase de maturité pour le dirigeant, puisque des valeurs différentes comme risque, croissance, profit et sécurité ressortent nettement. À ce propos, nous retrouvons l'opposition relative entre croissance et sécurité, celle-ci évoluant dans le même sens que l'âge au contraire de celle-là.

Pourtant, les études sur les cycles de vie considère le milieu de carrière des cadres comme une période de crise résultant d'un grand nombre de facteurs, tels que la diminution physique, la crise de la quarantaine, le ralentissement de la satisfaction professionnelle enregistrée jusqu'alors, etc. (Kets de Vries et Miller, 1985). Nous pouvons supposer que la fonction de direction éradique en partie les facteurs à la source de cette crise, notamment sur le plan professionnel.

Tableau 11

Relation de l'âge avec quelques variables

\begin{tabular}{lcccc}
\hline Variable considérée & $\begin{array}{c}31 \text { à } 40 \text { ans } \\
\mathbf{2 1 \%}\end{array}$ & $\begin{array}{c}\mathbf{4 1} \text { à } 50 \text { ans } \\
\mathbf{5 6 \%}\end{array}$ & $\begin{array}{c}\text { plus de } 51 \text { ans } \\
\mathbf{2 3} \%\end{array}$ & Test $\chi^{2}$ \\
\hline $\begin{array}{lccc}\text { Degré d'acceptation du risque } \\
\text { Importance accordée }\end{array}$ & +++ & +++ & ++ & n.s. \\
$\begin{array}{l}\text { à la croissance } \\
\text { Recherche du profit }\end{array}$ & ++++ & +++ & ++ & n.s. \\
$\begin{array}{l}\text { Besoin de sécurité } \\
\text { Objectif de leadership }\end{array}$ & + & ++++ & +++ & n.s. \\
$\quad$ dans la branche & + & +++ & +++ & n.s. \\
Objectif d'efficacité technique & ++++ & + & ++ & \\
\hline
\end{tabular}

* Non significatif au seuil de $5 \%$. 
Quant aux théories dites «de contenu » ${ }^{7}$, elles supposent, en fonction d'un développement à long terme, un besoin de sécurité important en début de carrière, un besoin d'accomplissement qui grandit jusqu'à 35 ans et décroît ensuite, tandis que les besoins d'affiliation et de pouvoir continuent à grandir lentement (Rojot et Bergmann, 1989). Ainsi, l'objectif de leadership dans la branche croissant avec l'âge pourrait se comprendre comme désir de pouvoir. Le besoin d'accomplissement ferait émerger une volonté de croissance plus forte chez les jeunes dirigeants que chez les plus âgés. En revanche, le besoin de sécurité en période de début de carrière a pu se manifester durant les années précédant l'accès à la fonction de direction ${ }^{8}$.

\section{Détention de capital}

Dans les PME, le capital est souvent détenu, dans sa totalité ou de façon majoritaire, par le chef d'entreprise ou par un groupe restreint de personnes. Par conséquent, il y a fréquemment symbiose entre le patrimoine social et celui du dirigeant, mais avec les risques que cela pose en cas de difficultè (Van Loye, 1991 ; Sainsaulieu, 1990). L'échantillon des dirigeants répondants dont nous disposons présente un ensemble conséquent de dirigeants non détenteurs $(52,94 \%)$, ce qui va permettre une comparaison avec les détenteurs de capital, les détenteurs majoritaires étant les plus nombreux.

Les détenteurs de capital déclarent accepter le risque, mais accordent plus d'importance au profit et à la sécurité. En revanche, la croissance semble être relativement plus importante pour les dirigeants non détenteurs. Les dirigeants non détenteurs accordent plus d'importance à l'indépendance financière et à l'égard des clients et fournisseurs, mais moins à l'égard des salariés. Les dirigeants détenteurs considèrent que les propriétaires devraient diriger leur affaires eux-mêmes ou en choisir les dirigeants. En revanche, la majorité des dirigeants non détenteurs déclarent que les propriétaires et le personnel devraient participer au choix des dirigeants ${ }^{9}$.

7. Les théories dites «de contenu» concernent l'ensemble des éléments moteurs du comportement issus de la personnalité de base. Ainsi, les théories de Freud et de ses successeurs ont permis l'émergence d'un certain nombre d'explications sur le comportement au travail et la motivation (Maslow, Alderfer, McClelland et Herzberg pour ne nommer qu'eux).

8. En moyenne, l'ancienneté dans la fonction de direction est de 9 années ; l'expérience dans le secteur d'activité est de 15 ans, et l'activité professionnelle est de 24 ans.

9. Test du $\chi^{2}$ égale 0,020 . 
TABleau 12

Relation de la détention du capital avec quelques variables

\begin{tabular}{lccc}
\hline Variable considérée & $\begin{array}{c}\text { Détenteurs } \\
\text { de capital }\end{array}$ & $\begin{array}{c}\text { Non détenteurs } \\
\text { de capital }\end{array}$ & Test de $\chi^{\mathbf{2}}$ \\
\hline Indépendance : & & & \\
- financière & +++ & ++ & 0,058 \\
- à l'égard des salariés & ++ & + & n.s. \\
- à l'égard des clients & + & +++ & 0,042 \\
et fournisseurs & & & \\
Importance accordée : & & +++ & n.s. \\
- à la croissance & ++ & ++ & n.s. \\
- au profit & +++ & ++ & 0,065 \\
- à la sécurité & +++ & +++ & n.s. \\
Acceptation du risque & ++ & & \\
\hline
\end{tabular}

* Non significatif au seuil de $10 \%$.

Ainsi, le dirigeant non détenteur présente un profil de manager centré sur la croissance avec des valeurs plus participatives que son homologue détenteur. Ce dernier correspond davantage à l'entrepreneur-propriétaire dépeint par la littérature où l'étude du fonctionnement et de l'évolution d'une PME nécessite de prendre en considération l'interaction qui existe entre l'entreprise et le dirigeant : la stratégie présente alors une dimension entrepreneuriale indivisée et une dimension patrimoniale (Sainsaulieu, 1990; McGuire, 1976).

\section{Formation et niveau d'expérience}

En premier lieu, le groupe de dirigeants NC (ne suivant pas comme objectif principal la croissance) a une constitution plus homogène quant au niveau de formation. La presque totalité d'entre eux $(95 \%)$ possède un niveau de formation supérieur dont la plupart en gestion. En revanche, pour les dirigeants $C$ (ayant la croissance comme objectif principal), le niveau de formation secondaire prend une place importante $(46,15 \%)$. L'ancienneté dans la fonction de dirigeant est en relation inverse avec l'objectif «croissance » (test du $\chi^{2}$ égale 0,001 ). De plus, il n'apparaît pas de liaison entre l'expérience acquise, quelles qu'en soient soit la nature et la croissance.

La formation, acquise initialement ou expérientiellement, ne semble pas favoriser le choix de la croissance. Ce résultat, à confirmer notamment par rapport à l'expérience, semble appuyer une thèse plus psychanalytique que comportementale, des théories de contenu plus que des théories de processus. Le choix de la croissance procéderait davantage de la projection, dans l'environnement entrepreneurial, d'un individu sécurisé donc apte au développement, plus que d'un savoir appliqué sur un terrain d'expérience. 


\section{Caractéristiques de l'entreprise}

Avec l'augmentation de l'âge de l'entreprise, l'intérêt pour l'objectif « croissance » semble diminuer. Les résultats montrent que les entreprises ayant comme objectif la croissance sont moins âgées (moyenne d'âge, 29 ans) que les entreprises ne suivant pas comme objectif la croissance (moyenne d'âge, 35 ans).

Les entreprises $C$ semblent recourir plus souvent à la sous-traitance et elles sont plus structurées en filiales que les entreprises NC. Dans cette situation, la croissance et la sous-traitance peuvent se comprendre comme des manifestations politiques de l'entreprise mère, plus que comme la volonté stricte du dirigeant.

Ainsi apparaît une cohérence qui serait à confirmer : l'entreprise qui opte pour la croissance serait dirigée par un manager salarié, non détenteur de capital plutôt qu'un entrepreneur-propriétaire ; elle suivrait la politique de l'entreprise mère ; elle aurait plus volontiers recours à la sous-traitance.

En conséquence, la croissance doit-elle être interprétée comme une résultante du contexte entrepreneurial ou comme la manifestation du système de valeurs d'un dirigeant accompli ?

\section{Conclusion}

Les résultats confirment les deux hypothèses formulées : le système de valeurs du dirigeant - socioculturel et entrepreneurial - permet de dresser le profil de celuici et se différencie en fonction du choix affirmé pour un objectif de croissance.

Les limites de l'étude réalisée proviennent du nombre relativement réduit de réponses et de la dimension subjective que revêt tout questionnaire. De ce fait, si la description des résultats permet de dresser un profil relativement cohérent du dirigeant, la nature et le sens des relations entre système de valeurs et croissance restent à structurer davantage. Enfin, une question centrale, qui concerne toutes les études à base de questionnaires, reste en suspens : existe-t-il une différence substantielle dans le profil de valeurs entre répondants et non répondants? Aussi, convient-il de rester très prudent quant à la généralisation des résultats énoncés.

La contribution de cette recherche réside essentiellement dans sa dimension exploratoire au regard du nombre restreint de recherches en la matière. Le profil du dirigeant, qui se dégage des résultats, est essentiellement caractérisé sur le plan sociologique par les valeurs religieuses, les valeurs relatives à la famille et à la personne et, sur le plan entrepreneurial, par les attitudes envers l'indépendance et la sécurité. Si les informations permettent l'établissement d'un profil relativement traditionnel dans la culture française et conforme à une vision classique de la fonction de direction dégagée par la littérature, l'apport spécifique ici réside dans la dimension 
de sérénité que manifestent la plupart des dirigeants répondants. Cette sérénité semble assise sur un climat de bonheur et un sentiment de libre arbitre.

Les valeurs des dirigeants suivant un objectif de croissance accentuent cette dernière tendance. Le choix de la croissance se manifeste davantage parmi les dirigeants bénéficiant d'un sentiment affirmé de bonheur, de confiance en soi, en autrui et en l'avenir. Il semble s'opposer à la recherche de la sécurité et de la protection, ne pas dépendre de la durée de l'expérience ou de la solidité de la formation initiale. De plus, le dirigeant qui dit choisir la croissance présente des caractéristiques managériales à orientation participative plus marquées que son homologue au profil plus traditionnel d'entrepreneur-propriétaire.

La relation entre bonheur et croissance qui se dégage des résultats confirme un certain nombre de thèses psychologiques en la matière, notamment le courant issu de la psychologie existentielle (Maslow, 1972 ; Rogers, 1966, etc.). Il ne faudrait pas pour autant ignorer les facteurs sociaux ou économiques, partiellement contrôlés dans l'enquête grâce au choix de l'échantillon.

Cependant, la prise en compte des variables de contexte éclairent sous un jour différent la croissance qui apparaît alors davantage comme la manifestation politique d'une organisation mère pilotant la PME.

Observer strictement un nombre suffisant de PME qui mettent en œuvre une stratégie de croissance permettra de dépasser ce questionnement apparemment alternatif : la croissance est-elle la volonté d'un entrepreneur accompli au sens psychologique ou le fruit d'un contexte entrepreneurial donné ?

\section{Bibliographie}

Allport, G.W., P.E. Vernon et G. Lindzey (1951, 1960), A Study of Values : A Scale for Measuring the Dominant Interests in Personality, Boston, Houghton Mifflin.

Aмıт, M. (1991), Les misères du patronat... Le monde des petites et moyennes entreprises industrielles et de leurs patrons, Paris, L'Harmattan, coll. Logiques sociales.

BAMBERGER, I. et E. GABELE (1980), «Les systèmes de valeurs des dirigeants et leur influence sur le comportement stratégique et la performance des entreprises ", Cahiers Stratégie et Organisation, Institut de gestion de Rennes, décembre.

BAmberger, I., A. Gueye, M. Moussayir et P. Robic (1986), «Dirigeants et stratégies des PMI-Rapport Stratos France 1 ", Cahier de recherche, Institut de gestion de Rennes.

BAYAD, M. et A. PARADAS (1995), «Les difficultés de recrutement en PME: recherche sur les déterminants organisationnels ", Actes du $\sigma^{e}$ congrès de l'AGRH, 23 et 24 novembre, Poitiers, p. 669-679. 
BAYAD, M. et J.L. HERMANN (1992), «Le financement de l'innovation dans les petites et moyennes entreprises industrielles : difficultés et comportement », Cahiers de recherche, Institut commercial de Nancy.

BENOîT, C. et M.D. Rousseau (1990), «La gestion des ressources humaines dans les petites et moyennes entreprises au Québec », Revue internationale P.M.E., vol. 3, $\mathrm{n}^{\mathrm{0}} 1$, p. 27-39.

Blais, A.R. et J.M. Toulouse (1990), «Les motivations des entrepreneurs : une étude empirique de 2278 fondateurs d'entreprises dans 14 pays », Revue internationale P.M.E., vol. 3, nos 3-4, p. 269-301.

BosWELL, J. (1971), «Corporate planning in small manufacturing firms », Journal of Business Policy, p. 49-62.

BURKE, M. (1991), Styles de pouvoir, Paris, Dunod.

CARrière, J.B. (1990), «La vision stratégique en contexte de PME : cadre théorique et étude empirique », Revue internationale P.M.E., vol. 3, nos 3-4, p. 301-327.

Chanlat, J.F. (1990), L'individu dans l'organisation. Les dimensions oubliées, Québec, Les Presses de l'Université Laval ; Ottawa, Éditions Eska.

Cossette, P. (1994), Cartes cognitives et organisations, Québec, Les Presses de l'Université Laval ; Ottawa, Éditions Eska.

ENGLAND, G.W. et R. LEE (1973), «Organization size as influence on perceived organizational goals : a comparative study among American, Japanese and Corean managers ", Organizational Behavior and Human Performance, vol. 9, $\mathrm{n}^{\circ} 1$, p. 48-59.

ENGLAND, G.W. (1975), The Manager and his Values : An International Perspective from the United States, Japan, Korea, India and Australia, Cambridge, Mass., Ballinger Publishing Co.

FERGUSON, M. (1981), Les enfants du Verseau : pour un nouveau paradigme, Paris, Calmann-Lévy.

Frank, H., D. Roessl, R.G. PlaschKa et H. Welsch (1991), «Valeurs guidant la planification des nouvelles entreprises : une comparaison internationale », Revue internationale P.M.E., vol. 4, no 1, p. 5-20.

Gartner, W.B., K.G. Shaver, E. Gatewood et J.A. Katz (1994), «Finding the entrepreneur in entrepreneurship», Entrepreneurship Theory and Practice, vol. 18, $\mathrm{n}^{\circ}$ 3, p. 5-9.

GAUTHEY, F., I. RATIU, I. RodGers et D. XARDEL (1988), Leaders sans frontières - Le défi des différences, Paris, McGraw-Hill, p. 12.

Guerra LeONE, N. et M. LE Berre (1990), «Une typologie des dirigeants de PME: l'exemple des dirigeants de PME du Nordeste brésilien », Économies et Sociétés, Série Sciences de Gestion, ${ }^{\circ}{ }^{0}$ 15, p. 7-37. 
JULIEN, P.A. et M. MARCHESNAY (1988), La petite entreprise : principes d'économie et de gestion, Paris, Vuibert Gestion.

JULIEN, P.A. et M. MARChESNAY (1992), «Des procédures aux processus stratégiques dans les PME », A. Noël (dir.), Perspectives en management stratégique, tome I, Paris, Economica Gestion, p. 97-129.

KASHEFI-ZIHAGH, M. (1970), An Empirical Investigation of the Relationship between Value Systems and Organizational Effectiveness, Mémoire de DBA non publié, Michigan State University, Ann Arbor.

KeTS DE VRIES, M.F.R. et D. MiLleR (1985), L'entreprise névrosée, Paris, McGraw-Hill.

Kostenbaum, P. (1989), Socrate et le Business. Les conseils d'un philosophe aux dirigeants d'entreprise, Paris, InterÉditions.

LARÇON, J.P. et R. REITTER (1971), Structures du pouvoir et identité de l'entreprise, Paris, Nathan Sociologies, p. 45-47.

LAUfER, J. (1975), «Comment on devient entrepreneur», Revue Française de Gestion, $\mathrm{n}^{\circ} 2$, novembre, p. $11-27$.

LECOINTRE, G. (1977), Essai de typologie des petites entreprises familiales, thèse de doctorat, Université de Paris-Dauphine.

Lefebvre, E. (1992), «Profil distinctif des dirigeants de P.M.E. innovatrices », Revue internationale P.M.E., $3^{\mathrm{e}}$ trimestre, p. 7-26.

LORRAIN, J. et L. DUSSAULT (1988), «Les entrepreneurs artisans et opportunistes : une comparaison de leurs comportements de gestion", Revue internationale P.M.E., vol. $1, \mathrm{n}^{\mathrm{0}} 3$, p. 158-176.

MAHÉ DE BoISLANDELLE, H. (1988), Gestion des ressources humaines dans les PME, Paris, Economica.

MARCHESNAY, M. (1988), «L'externalisation des services aux entreprises, le cas de PME montpelliéraines ", Revue de l'économie méridionale, vol. 35, $\mathrm{n}^{\text {os }} 2-3$, p. 65-76.

MARMUSE, C. (1992), Politique générale : langages, intelligence, modèles et choix stratégiques, Paris, Economica, p. 241, 463-485.

MaSLOW, A.H. (1972), Vers une psychologie de l'être, Paris, Fayard.

MCGUIRE, J. (1976), «The small entreprise in economic and organisation theory », Journal of Contemporary Business, printemps, p. 115-138.

MiLlER, D. et J.M. Toulouse (1986), « Chief executive personality and corporate strategy and structure in small firms», Management Science, vol. 32, novembre, p. 1389-1409.

Miller, D., C. Droge et J.M. Toulouse (1988), «Strategic process and content as mediators between organizational context and structure ", Academy of Management Journal, vol. 31, septembre, p. 544-569. 
MinTZBERG, H. (1990), Le management-Voyage au centre des organisations, Paris, Éditions d'Organisation; Montréal, Agence d'Arc.

Morgan, G. (1989), Images de l'organisation, Québec, Les Presses de l’Université Laval ; Ottawa, Éditions Eska.

Moss KANTER, R. (1984), The Change Masters, New York, Simon and Schuster.

NeWComb, M.T., H.R. TURner et E.P. CONVERSE (1970), Manuel de psychologie sociale, Paris, Presses universitaires de France.

Pras, B. et E. RouX (1990), «Variables individuelles interculturelles et stratégie de croissance des PME », dans F. Gauthey et D. Xardel (dir.), Management interculturelMythes et réalités (I)-Modes et modèles (II), Paris, Economica, p. 13-38.

QUIVY, R. et L. VAN CAMPENHOUDT (1988), Manuel de recherche en sciences sociales, Paris, Dunod.

REITTER, R. et al. (1991), Cultures d'entreprise-Étude sur les conditions de réussite du changement, Paris, Vuibert Gestion.

RogERS, C. (1966), Le développement de la personne, Paris, Dunod.

Rojot, J. et A. Bergmann (1989), Comportement et organisation, Paris, Vuibert Gestion.

Rokeach, M. (1973), The Nature of Human Values, New York, Free Press.

Sainsaulieu, R. (1990), L'entreprise une affaire de société, Paris, Presses de la Fondation nationale des sciences politiques.

StOETZEL, J. (1983), Les valeurs du temps présent : une enquête européenne, Paris, Presses universitaires de France.

STRATEGOR (1991), Stratégie, structure, décision, identité, politique générale d'entreprise, Paris, InterÉditions.

STRATOS (1987), « Strategic orientations of small and medium-sized enterprises », Institute Report 87-01, European Institute for Advanced Studies in Management.

VAN Loye, G. (1991), «Organisation du pouvoir et financement des P.M.E. », Direction et Gestion des Entreprises, nos 132-133, décembre, p. 47-53.

VIGNON, Ch. et F. ZADDEM (1989), "Changement de culture ou culture du changement », Cahiers de recherche, $\mathrm{n}^{\circ} 3$, IAE de Lille. 